TRANSACTIONS OF THE

AMERICAN MATHEMATICAL SOCIETY

Volume 350, Number 3, March 1998, Pages 1225-1247

S 0002-9947(98)02080-7

\title{
THE POSSIBLE ORDERS OF SOLUTIONS OF LINEAR DIFFERENTIAL EQUATIONS WITH POLYNOMIAL COEFFICIENTS
}

\author{
GARY G. GUNDERSEN, ENID M. STEINBART, AND SHUPEI WANG
}

ABSTRACT. We find specific information about the possible orders of transcendental solutions of equations of the form $f^{(n)}+p_{n-1}(z) f^{(n-1)}+\cdots+p_{0}(z) f=$ 0 , where $p_{0}(z), p_{1}(z), \ldots, p_{n-1}(z)$ are polynomials with $p_{0}(z) \not \equiv 0$. Several examples are given.

\section{INTRODUCTION}

For $n \geq 2$, consider a linear differential equation of the form

$$
f^{(n)}+p_{n-1}(z) f^{(n-1)}+\cdots+p_{0}(z) f=0,
$$

where $p_{0}(z), \ldots, p_{n-1}(z)$ are polynomials with $p_{0}(z) \not \equiv 0$. It is well known that every solution $f$ of equation (1.1) is an entire function of finite rational order; see [7], [8], [3, pp. 199-209], [6, pp. 106-108], [9, pp. 65-67].

For equation (1.1), set

$$
\lambda=1+\max _{0 \leq k \leq n-1} \frac{\operatorname{deg} p_{k}}{n-k} .
$$

Let $\rho(f)$ denote the order of an entire function $f$. It is known [4, p. 127] that for any solution $f$ of (1.1),

$$
\rho(f) \leq \lambda .
$$

Wittich obtained the following result.

Theorem A [8], [9, pp. 65-67]. For a given equation of the form (1.1), there exists a set of positive rational numbers $\chi_{1}, \chi_{2}, \ldots, \chi_{k}$, where $k \leq n$, such that if $f$ is any transcendental solution of equation (1.1), then

$$
\rho(f)=\chi_{j}
$$

for some $j, 1 \leq j \leq k$.

In his proof of Theorem A, Wittich used the method of Frobenius, the WimanValiron theory, the theory of algebraic functions, and the Newton-Puiseux diagram, where the rational numbers $\chi_{1}, \chi_{2}, \ldots, \chi_{k}$ are determined from the Newton-Puiseux diagram. Helmrath and Nikolaus [2] and Jank and Volkmann [3, pp. 199-209] also gave a proof of Theorem A, each on a more general equation than (1.1), where their proofs use the Wiman-Valiron theory, the theory of algebraic functions, and the Newton-Puiseux diagram.

Received by the editors October 11, 1995 and, in revised form, July 6, 1996.

1991 Mathematics Subject Classification. Primary 34A20; Secondary 30D35.

(C)1998 American Mathematical Society 
Theorem 1 in $\S 2$ gives a list of positive rational numbers that includes all the possible orders of transcendental solutions of equation (1.1). This list of rational numbers is obtained from simple arithmetic with the degrees of the polynomial coefficients in (1.1). We do not appeal to the Newton-Puiseux diagram to obtain this list of rational numbers or to prove Theorem 1.

Some natural questions can be asked. For example:

(i) What is the maximum number of possible distinct orders of transcendental solutions of a given equation of the form (1.1)?

(ii) Consider (1.3). Is the upper bound $\lambda$ always reached? In other words, for any given equation of the form (1.1), does there always exist a solution $f$ of (1.1) that satisfies $\rho(f)=\lambda$, where $\lambda$ is the constant in (1.2)?

(iii) What is the smallest possible sum of the orders of a fundamental set of solutions of a given equation of the form (1.1)?

(iv) What is the maximum number of linearly independent polynomial solutions that an equation of the form (1.1) can possess?

In this paper we answer these four questions, and we also give related results. Several examples are given to illustrate our results.

\section{Statement of Results}

Consider equation (1.1). For convenience, set $d_{j}=\operatorname{deg} p_{j}$ if $p_{j} \not \equiv 0$ and $d_{j}=-\infty$ if $p_{j} \equiv 0,0 \leq j \leq n-1$.

We define a strictly decreasing finite sequence of non-negative integers

$$
s_{1}>s_{2}>\cdots>s_{p} \geq 0
$$

in the following manner. We choose $s_{1}$ to be the unique integer satisfying

$$
\frac{d_{s_{1}}}{n-s_{1}}=\max _{0 \leq k \leq n-1} \frac{d_{k}}{n-k} \quad \text { and } \quad \frac{d_{s_{1}}}{n-s_{1}}>\frac{d_{k}}{n-k} \quad \text { for all } 0 \leq k<s_{1} .
$$

Then given $s_{j}, j \geq 1$, we define $s_{j+1}$ to be the unique integer satisfying

$$
\begin{aligned}
& \frac{d_{s_{j+1}}-d_{s_{j}}}{s_{j}-s_{j+1}}=\max _{0 \leq k<s_{j}} \frac{d_{k}-d_{s_{j}}}{s_{j}-k}>-1 \text { and } \\
& \frac{d_{s_{j+1}}-d_{s_{j}}}{s_{j}-s_{j+1}}>\frac{d_{k}-d_{s_{j}}}{s_{j}-k} \text { for all } 0 \leq k<s_{j+1} .
\end{aligned}
$$

For a certain $p$, the integer $s_{p}$ will exist, but the integer $s_{p+1}$ will not exist, and then the sequence $s_{1}, s_{2}, \ldots, s_{p}$ terminates with $s_{p}$. Obviously, $p \leq n$, and we also see that (2.1) holds.

Correspondingly, define for $j=1,2, \ldots, p$,

$$
\alpha_{j}=1+\frac{d_{s_{j}}-d_{s_{j-1}}}{s_{j-1}-s_{j}}
$$

where we set

$$
s_{0}=n \quad \text { and } \quad d_{s_{0}}=d_{n}=0 .
$$

From (2.3) and (2.4), we observe that $\alpha_{j}>0$ for each $j, 1 \leq j \leq p$.

We mention that the integers $s_{1}, s_{2}, \ldots, s_{p}$ in (2.1) can also be expressed in the following manner:

$$
s_{1}=\min \left\{j: \frac{d_{j}}{n-j}=\max _{0 \leq k \leq n-1} \frac{d_{k}}{n-k}\right\}
$$


and given $s_{j}, j \geq 1$, we have

$$
s_{j+1}=\min \left\{i: \frac{d_{i}-d_{s_{j}}}{s_{j}-i}=\max _{0 \leq k<s_{j}} \frac{d_{k}-d_{s_{j}}}{s_{j}-k}>-1\right\} .
$$

We prove the following result.

Theorem 1. For equation (1.1), the following conclusions hold:

(i) If $f$ is a transcendental solution of (1.1), then $\rho(f)=\alpha_{j}$ for some $j, 1 \leq j \leq$ $p$.

(ii) If $s_{1} \geq 1$ and $p \geq 2$, then the following inequalities hold:

$$
\alpha_{1}>\alpha_{2}>\cdots>\alpha_{p} \geq \frac{1}{s_{p-1}-s_{p}} \geq \frac{1}{s_{1}-s_{p}} \geq \frac{1}{s_{1}} .
$$

(iii) If $s_{1}=0$, then any nontrivial solution $f$ of (1.1) satisfies $\rho(f)=1+d_{0} / n$.

Theorem 1(iii) is known [9, Chap. V]. We will, however, give a new proof of this result by using sharp estimates of logarithmic derivatives; see the remark at the end of $\S 6$.

Regarding Theorem 1(ii), in the case when $s_{1} \geq 1$ and $p=1$, we obtain from Theorem 1(i), (2.2), (2.4), and (2.5) that any transcendental solution $f$ of (1.1) satisfies $\rho(f)=\lambda$, where $\lambda$ is the constant in (1.2).

We mention that Pöschl [5] gave a detailed analysis of the possible orders of transcendental solutions of (1.1) in the case when $n=3$, and Theorem 1 gives an improvement of this result of Pöschl.

Since $p \leq n$ in (2.4), a corollary of Theorem 1 is the known result that there can exist at most $n$ distinct possible orders of transcendental solutions of equation (1.1); see Theorem A. However, Theorem 1 yields more than this result. We observe from (2.2) and (2.3) that the integer $p$ satisfies $p \leq s_{1}+1$. Then from Theorem 1 and the construction of $\alpha_{j}$ in (2.4), we deduce the following result.

Corollary 1. There can exist at most $s_{1}+1$ distinct orders of transcendental solutions of (1.1). Furthermore, if an equation of the form (1.1) possesses $s_{1}+1$ transcendental solutions that have $s_{1}+1$ distinct orders, then these $s_{1}+1$ orders must be the following numbers:

$$
1+\frac{d_{s_{1}}}{n-s_{1}}, 1+d_{s_{1}-1}-d_{s_{1}}, 1+d_{s_{1}-2}-d_{s_{1}-1}, \ldots, 1+d_{0}-d_{1} .
$$

Consequently, in this case, $d_{s_{1}} \leq d_{s_{1}-1} \leq \cdots \leq d_{1} \leq d_{0}$.

Examples 2 through 5 in $\S 8$ illustrate the sharpness of Corollary 1. In the special case when $s_{1}=1$, it follows from Corollary 1 and Theorem 1 that there can exist at most two distinct orders of transcendental solutions of (1.1), and these two possible orders are $1+d_{1} /(n-1)$ and $1+d_{0}-d_{1}$.

Next, from $(2.2)$ we see that

$$
s_{1} \leq n-1 \text {. }
$$

Combining (2.6) with Theorem 1 yields the following result.

Corollary 2. Every transcendental solution $f$ of (1.1) satisfies

$$
\rho(f) \geq \frac{1}{n-1} .
$$


Wittich [8], [9, pp. 65-68] proved Corollary 2, and he also gave an example to indicate that Corollary 2 is sharp for all $n \geq 2$ (see the remark at the end of Example 1 in §8). In his proof of Corollary 2, Wittich used the method of Frobenius and the Newton-Puiseux diagram, which we do not appeal to here. In Example 1 in $\S 8$, we exhibit an equation of the form (1.1) where $n=3$, which possesses a special contour integral solution $f=G(z)$ satisfying $\rho(G)=1 / 2$, which gives equality in (2.7). Of course all nontrivial solutions of equations of the form (1.1) with constant coefficients have order one, which gives equality in (2.7) when $n=2$.

We prove the following result.

Theorem 2. For any $j=1,2, \ldots, p$, there can exist at most $s_{j}$ linearly independent solutions $f$ of (1.1) satisfying $\rho(f)<\alpha_{j}$.

Examples 2 through 6 in $\S 8$ illustrate the sharpness of Theorem 2.

Note that $\alpha_{1}=\lambda$, where $\alpha_{1}$ is defined in (2.4) and $\lambda$ is the constant in (1.2). From (1.3), the order of any solution of (1.1) cannot be greater than $\lambda$. From (2.6) and Theorem 2 (with $j=1$ ), we obtain the following result, which says that this maximum possible order $\lambda$ is always reached.

Corollary 3. For any given equation of the form (1.1), there must exist a solution of (1.1) that satisfies $\rho(f)=\lambda$, where $\lambda$ is the constant in (1.2).

Thus from (1.3) and Theorem 1, Corollary 3 shows that there always exists a solution of equation (1.1) that has the maximum possible order $\lambda=\alpha_{1}$. This shows, among other things, that it is not possible for an equation of the form (1.1) to have only polynomials for solutions. Moreover, we observe from Theorem 1 that any solution $f \not \equiv 0$ of $(1.1)$ satisfying $\rho(f)<\alpha_{p}$ must be a polynomial. Combining this with Theorem 2 yields the following result.

Corollary 4. There can exist at most $s_{p}$ linearly independent polynomial solutions of (1.1).

Corollary 4 is sharp; see Example 6 in $\S 8$.

Wittich proved the following result.

Theorem B [8]. Suppose that every nontrivial solution of (1.1) is transcendental. If $\left\{f_{1}, f_{2}, \ldots, f_{n}\right\}$ is any fundamental set of solutions of (1.1), then

$$
\sum_{k=1}^{n} \rho\left(f_{k}\right) \geq n
$$

We can improve Theorem B by appealing to Theorem 2. Specifically, suppose that $\left\{f_{1}, f_{2}, \ldots, f_{n}\right\}$ is any fundamental set of solutions of equation (1.1), where we allow the possibility that an $f_{k}$ might be a polynomial. From Theorem 2 and Corollary 4, it can be deduced that

$$
\sum_{k=1}^{n} \rho\left(f_{k}\right) \geq\left(n-s_{1}\right) \alpha_{1}+\left(s_{1}-s_{2}\right) \alpha_{2}+\cdots+\left(s_{p-1}-s_{p}\right) \alpha_{p}+s_{p} \cdot 0 .
$$

From (2.4), we obtain that the right side of (2.9) equals $n+d_{s_{p}}-s_{p}$, and so

$$
\sum_{k=1}^{n} \rho\left(f_{k}\right) \geq n+d_{s_{p}}-s_{p}
$$


Now $d_{s_{p}}-s_{p} \geq d_{0}$, because if $s_{p}=0$, then $d_{s_{p}}-s_{p}=d_{0}$, while if $s_{p} \geq 1$, then from (2.3) and the fact that $s_{p}$ is the last element in the sequence $s_{1}, s_{2}, \ldots, s_{p}$, we obtain $\left(d_{0}-d_{s_{p}}\right) /\left(s_{p}-0\right) \leq-1$. Combining this with $(2.10)$ gives the following result.

Corollary 5. If $\left\{f_{1}, f_{2}, \ldots, f_{n}\right\}$ is any fundamental set of solutions of (1.1), then

$$
\sum_{k=1}^{n} \rho\left(f_{k}\right) \geq n+d_{0}
$$

Corollary 5 is sharp. This is illustrated by Examples 2 through 6 in $\S 8$ and by the situation in Theorem 1(iii). Corollary 5 is an improvement of Theorem B because (2.11) improves (2.8), and also because nontrivial polynomial solutions of (1.1) are allowed.

In $\S \S 3-7$ we give the proofs of Theorems 1 and 2 . In $\S 8$ we give several examples to illustrate the sharpness of our results, and also to exhibit some possibilities that can occur.

We mention that we prove Theorem 1(ii) first (in §3), because we use Theorem 1(ii) in the proof of Lemma 4.2 in $\S 4$, and Lemma 4.2 is used in the proof of Theorem $1(\mathrm{i})$ in $\S 5$.

\section{Proof of Theorem 1(ii)}

We first prove $\alpha_{1}>\alpha_{2}>\cdots>\alpha_{p}$. From (2.1), (2.2), (2.3), and (2.5), we obtain, for any $j=1,2, \ldots, p-1$,

$$
s_{j}>s_{j+1} \quad \text { and } \quad \frac{d_{s_{j}}-d_{s_{j-1}}}{s_{j-1}-s_{j}}>\frac{d_{s_{j+1}}-d_{s_{j-1}}}{s_{j-1}-s_{j+1}},
$$

which yields

$$
-d_{s_{j}} s_{j+1}-d_{s_{j-1}}\left(s_{j}-s_{j+1}\right)>d_{s_{j+1}}\left(s_{j-1}-s_{j}\right)-d_{s_{j}} s_{j-1} .
$$

Adding $d_{s_{j}} s_{j}$ to both sides of (3.1) gives

$$
\begin{aligned}
\left(d_{s_{j}}-d_{s_{j-1}}\right)\left(s_{j}-s_{j+1}\right) & >\left(d_{s_{j+1}}-d_{s_{j}}\right)\left(s_{j-1}-s_{j}\right), \\
\frac{d_{s_{j}}-d_{s_{j-1}}}{s_{j-1}-s_{j}} & >\frac{d_{s_{j+1}}-d_{s_{j}}}{s_{j}-s_{j+1}} .
\end{aligned}
$$

From the definition of $\alpha_{j}$ in (2.4), we obtain immediately from (3.2) that $\alpha_{j}>\alpha_{j+1}$. This proves that

$$
\alpha_{1}>\alpha_{2}>\cdots>\alpha_{p}
$$

From (2.1) we have

$$
\frac{1}{s_{p-1}-s_{p}} \geq \frac{1}{s_{1}-s_{p}} \geq \frac{1}{s_{1}}
$$

and so to complete the proof of Theorem 1(ii), we need only to prove that

$$
\alpha_{p} \geq \frac{1}{s_{p-1}-s_{p}} .
$$

From (2.3) we obtain

$$
\frac{d_{s_{p}}-d_{s_{p-1}}}{s_{p-1}-s_{p}}>-1,
$$




$$
d_{s_{p}}-d_{s_{p-1}}+s_{p-1}-s_{p}>0 .
$$

Since the left side of (3.6) is an integer, we have $d_{s_{p}}-d_{s_{p-1}}+s_{p-1}-s_{p} \geq 1$. Hence from (2.4), we see that (3.5) holds. By combining (3.5), (3.4), and (3.3), we obtain Theorem 1(ii).

\section{Lemmas For the Proof of Theorem 1(i)}

We use the three lemmas in this section in the proof of Theorem 1(i).

Lemma 4.1. For any fixed $j=0,1, \ldots, p-1$, let $\alpha$ be any real number satisfying $\alpha>\alpha_{j+1}$, and let $k$ be any integer satisfying $0 \leq k<s_{j}$. Then

$$
n-k+d_{k}+k \alpha<n-s_{j}+d_{s_{j}}+s_{j} \alpha .
$$

Proof. Since $n-k+d_{k}+k \alpha=\left(n-s_{j}+d_{s_{j}}+s_{j} \alpha\right)+\alpha\left(k-s_{j}\right)+d_{k}-d_{s_{j}}+s_{j}-k$, we obtain

(4.2) $n-k+d_{k}+k \alpha<\left(n-s_{j}+d_{s_{j}}+s_{j} \alpha\right)+\alpha_{j+1}\left(k-s_{j}\right)+d_{k}-d_{s_{j}}+s_{j}-k$.

Now from the definition of $\alpha_{j+1}$ in (2.4), we obtain

$$
\alpha_{j+1}\left(k-s_{j}\right)+d_{k}-d_{s_{j}}+s_{j}-k=\left(k-s_{j}\right)\left(\frac{d_{s_{j+1}}-d_{s_{j}}}{s_{j}-s_{j+1}}-\frac{d_{k}-d_{s_{j}}}{s_{j}-k}\right) .
$$

Since $0 \leq k<s_{j}$, it follows from the definition of $s_{j+1}$ in (2.2) and (2.3) that

$$
\frac{d_{s_{j+1}}-d_{s_{j}}}{s_{j}-s_{j+1}} \geq \frac{d_{k}-d_{s_{j}}}{s_{j}-k}
$$

From (4.4) and (4.3) we obtain

$$
\alpha_{j+1}\left(k-s_{j}\right)+d_{k}-d_{s_{j}}+s_{j}-k \leq 0 .
$$

Then (4.1) follows from (4.2) and (4.5).

Lemma 4.2. For any fixed $j=1,2, \ldots, p$, let $\alpha$ be any real number satisfying $\alpha<$ $\alpha_{j}$, and let $k$ be any integer satisfying $s_{j}<k \leq n$. Then

$$
n-k+d_{k}+k \alpha<n-s_{j}+d_{s_{j}}+s_{j} \alpha .
$$

Proof. We consider two separate cases.

Case (i). Suppose that $s_{j}<k \leq s_{j-1}$.

This case uses an argument similar to the proof of Lemma 4.1. As in the proof of Lemma 4.1, we have

$$
n-k+d_{k}+k \alpha<\left(n-s_{j}+d_{s_{j}}+s_{j} \alpha\right)+\alpha_{j}\left(k-s_{j}\right)+d_{k}-d_{s_{j}}+s_{j}-k .
$$

Then from the definition of $\alpha_{j}$ in (2.4), we obtain

$$
\alpha_{j}\left(k-s_{j}\right)+d_{k}-d_{s_{j}}+s_{j}-k=\left(k-s_{j}\right) \frac{d_{s_{j}}-d_{s_{j-1}}}{s_{j-1}-s_{j}}+d_{k}-d_{s_{j}} .
$$

If $k=s_{j-1}$, then the right side of (4.8) equals zero. Then (4.6) follows from (4.7).

On the other hand, if $s_{j}<k<s_{j-1}$, then from the definition of $s_{j}$ in $(2.2)$ and (2.3), we obtain

$$
\left(k-s_{j}\right) \frac{d_{s_{j}}-d_{s_{j-1}}}{s_{j-1}-s_{j}}+d_{k}-d_{s_{j}}=\left(k-s_{j-1}\right)\left(\frac{d_{s_{j}}-d_{s_{j-1}}}{s_{j-1}-s_{j}}-\frac{d_{k}-d_{s_{j-1}}}{s_{j-1}-k}\right)
$$

$$
\leq 0 .
$$


Combining (4.9), (4.8), and (4.7) gives (4.6). This proves Lemma 4.2 for Case (i).

Case (ii). Suppose that $s_{j-1}<k \leq n$.

Since $s_{j}<s_{j-1}<\cdots<s_{1}<s_{0}=n$ and $s_{j-1}<k \leq n$, it follows that $j \geq 2$ and there exists an integer $m, 1 \leq m \leq j-1$, such that $s_{j-m}<k \leq s_{j-m-1}$. Also, from Theorem 1(ii), which we proved in $\S 3$, we have

$$
\alpha_{j}<\alpha_{j-1}<\cdots<\alpha_{j-m} .
$$

Since $\alpha<\alpha_{j}$, we have $\alpha<\alpha_{j-m}$. Hence we can apply Case (i) to obtain that

$$
n-k+d_{k}+k \alpha<n-s_{j-m}+d_{s_{j-m}}+s_{j-m} \alpha .
$$

Now from successive applications of Case (i), we obtain the following inequalities:

$$
\begin{aligned}
& n-s_{j-1}+d_{s_{j-1}}+s_{j-1} \alpha<n-s_{j}+d_{s_{j}}+s_{j} \alpha \quad \text { for } \quad \alpha<\alpha_{j}, \\
& n-s_{j-2}+d_{s_{j-2}}+s_{j-2} \alpha<n-s_{j-1}+d_{s_{j-1}}+s_{j-1} \alpha \quad \text { for } \quad \alpha<\alpha_{j-1} \text {, } \\
& \text {.. } \ldots \quad \ldots \\
& n-s_{j-m}+d_{s_{j-m}}+s_{j-m} \alpha<n-s_{j-m+1}+d_{s_{j-m+1}}+s_{j-m+1} \alpha \\
& \text { for } \quad \alpha<\alpha_{j-m+1} \text {. }
\end{aligned}
$$

It follows from (4.10) that all of the above inequalities hold for $\alpha<\alpha_{j}$. Therefore, by combining these inequalities with (4.11), we obtain (4.6). This proves Case (ii), and completes the proof of Lemma 4.2 .

Lemma 4.3. Let $\alpha>0$. Then for any integer $k$ satisfying $0 \leq k<s_{p}$, we have

$$
n-k+d_{k}+k \alpha<n-s_{p}+d_{s_{p}}+s_{p} \alpha .
$$

Proof. Since $s_{p}$ is the last element in the sequence $s_{1}, s_{2}, \ldots, s_{p}$, it follows from the construction of $s_{p}$ in (2.2) and (2.3) that for any $k<s_{p}$,

$$
\frac{d_{k}-d_{s_{p}}}{s_{p}-k} \leq-1 \text {. }
$$

This gives $d_{k}-k \leq d_{s_{p}}-s_{p}$. Since $k \alpha<s_{p} \alpha$, we obtain (4.12).

\section{Proof of Theorem 1(i)}

Let $f$ be a transcendental (entire) solution of (1.1) with order $\rho(f)$. The statements in (5.1), (5.2), and (5.3) below are well known; see [3, pp. 199-209], [6, pp. 105-108], and [9, pp. 65-67]. We have

$$
0<\rho(f)<\infty .
$$

Furthermore, if $V(r)$ denotes the central index of $f$, then

$$
V(r)=(1+o(1)) C r^{\alpha}
$$

as $r \rightarrow \infty$, where $\alpha=\rho(f)$ and $C$ is a positive constant. In addition, from the Wiman-Valiron theory it follows that there exists a set $E_{0} \subset(0, \infty)$ that has finite logarithmic measure, such that for all $q=1,2, \ldots, n$ we have

$$
\frac{f^{(q)}\left(z_{r}\right)}{f\left(z_{r}\right)}=(1+o(1))\left(\frac{V(r)}{z_{r}}\right)^{q}
$$

as $r \rightarrow \infty, r \notin E_{0}$, where $z_{r}$ is a point on the circle $|z|=r$ that satisfies $\left|f\left(z_{r}\right)\right|=$ $M(r, f)$. Here $M(r, f)$ denotes the usual maximum modulus function: $M(r, f)=$ $\max _{|z|=r}|f(z)|, 0<r<\infty$. 
Now in equation (1.1), for each $k, 0 \leq k \leq n-1$, let $b_{k}$ denote the leading coefficient of the polynomial $p_{k}(z)$, and set $a_{k}=C^{k}\left|b_{k}\right|$, where $C>0$ is the constant in (5.2). Also set $a_{n}=C^{n}$. We now divide equation (1.1) by $f$, and then substitute (5.3) and (5.2) into (1.1). This yields an equation whose right side is zero and whose left side consists of a sum of $n+1$ terms whose absolute values are asymptotic (as $r \rightarrow \infty, r \notin E_{0}$ ) to the following $n+1$ terms:

$$
a_{n} r^{n \alpha}, a_{n-1} r^{1+d_{n-1}+(n-1) \alpha}, \cdots, a_{k} r^{n-k+d_{k}+k \alpha}, \cdots, a_{0} r^{n+d_{0}} .
$$

Now from (1.2) and (1.3), the order of any solution of (1.1) is at most $\lambda$, and $\lambda=\alpha_{1}$ from (2.4) and (2.2). Thus $\alpha \leq \alpha_{1}$.

Now suppose that $\alpha_{j+1}<\alpha<\alpha_{j}$ for some $j=1,2, \ldots, p-1$. Then from Lemma 4.1 and Lemma 4.2 , we obtain that

$$
n-k+d_{k}+k \alpha<n-s_{j}+d_{s_{j}}+s_{j} \alpha \quad \text { for any } \quad k \neq s_{j} .
$$

But from inspection of (5.5) and (5.4), we see that there will exist exactly one dominant term (as $r \rightarrow \infty, r \notin E_{0}$ ) in (5.4). Specifically, there exists exactly one term in (5.4) with exponent $n-s_{j}+d_{s_{j}}+s_{j} \alpha$, where $a_{s_{j}} \neq 0$, such that the exponent $n-s_{j}+d_{s_{j}}+s_{j} \alpha$ is greater than all the other exponents of the terms in (5.4). This is impossible.

On the other hand, suppose that $\alpha<\alpha_{p}$. Then from Lemma 4.2 and Lemma 4.3 , we obtain

$$
n-k+d_{k}+k \alpha<n-s_{p}+d_{s_{p}}+s_{p} \alpha \quad \text { for any } \quad k \neq s_{p}
$$

Again, by the same reasoning, (5.6) is impossible, because otherwise (5.4) would have exactly one dominant term as $r \rightarrow \infty, r \notin E_{0}$.

Therefore, the only admissible values for $\alpha$, the order of $f$, are $\alpha_{1}, \alpha_{2}, \ldots, \alpha_{p}$. This proves Theorem 1(i).

\section{LEMmas FOR The PROOF OF TheOREM 2}

For the rest of the paper we make the following two conventions: (i) A meromorphic function will always be meromorphic in the whole complex plane. (ii) We will let $E=E_{0} \cup[0,1]$, where $E_{0}$ is a set in $0<r<\infty$ that has finite logarithmic measure, and the set $E$ may not necessarily be the same set each time it appears.

We also mention that the definition of the order of a meromorphic function is the standard definition from Nevanlinna theory (see [4, p. 24]), and this definition generalizes the definition of the order of an entire function. As with entire functions, we again use $\rho(f)$ to denote the order of a meromorphic function $f$.

Our proof of Theorem 2 will consist of combining the standard method of reduction of order for linear differential equations with the following result.

Lemma 6.1 [1]. Let $f \not \equiv 0$ be a meromorphic function of finite order $\beta$, and let $k \geq 1$ be an integer. Then for any given $\varepsilon>0$, we have

$$
\left|\frac{f^{(k)}(z)}{f(z)}\right| \leq|z|^{k(\beta-1)+\varepsilon}, \quad|z| \notin E .
$$

We remark that the estimate $(6.1)$ is sharp in the sense that we cannot replace (6.1) with the statement ' $\left|f^{(k)}(z) / f(z)\right| \leq C|z|^{k(\beta-1)}$, where $C>0$ is some constant' (see $[1, \S 9]$ ). We need this sharpness in our proof of Theorem 2 , and also in our new proof of Theorem 1(iii).

Lemmas 6.2 to 6.5 below are concerned with the method of reduction of order. 
Lemma 6.2. Let $f_{1}, f_{2}, \ldots, f_{N}(N \geq 2)$ be $N$ linearly independent meromorphic functions. Set $h_{j}=\left(f_{j+1} / f_{1}\right)^{\prime}$ for $j=1,2, \ldots, N-1$. Then $h_{1}, h_{2}, \ldots, h_{N-1}$ are $N-1$ linearly independent meromorphic functions.

Proof. Suppose that $h_{1}, h_{2}, \ldots, h_{N-1}$ are linearly dependent. Then there exist $N-1$ constants $c_{1}, c_{2}, \ldots, c_{N-1}$, which are not all zero, such that

$$
c_{1} h_{1}+c_{2} h_{2}+\cdots+c_{N-1} h_{N-1} \equiv 0,
$$

i.e.,

$$
\frac{d}{d z}\left(\frac{c_{1} f_{2}+c_{2} f_{3}+\cdots+c_{N-1} f_{N}}{f_{1}}\right) \equiv 0
$$

It follows that there exists some constant $c_{0}$ such that

$$
c_{0} f_{1}+c_{1} f_{2}+c_{2} f_{3}+\cdots+c_{N-1} f_{N} \equiv 0 .
$$

Hence, $f_{1}, f_{2}, \ldots, f_{N}$ are linearly dependent, which contradicts our assumption.

Lemma 6.3. Let $f$ and $g$ be two linearly independent meromorphic solutions of an equation of the form

$$
y^{(n)}+A_{n-1}(z) y^{(n-1)}+\cdots+A_{1}(z) y^{\prime}+A_{0}(z) y=0,
$$

where $A_{0}(z), A_{1}(z), \ldots, A_{n-1}(z)$ are meromorphic functions. Set $u=(f / g)^{\prime}$. Then $y=u(z)$ satisfies the equation

$$
y^{(n-1)}+B_{n-2}(z) y^{(n-2)}+\cdots+B_{1}(z) y^{\prime}+B_{0}(z) y=0,
$$

where

$$
B_{j}(z)=\sum_{k=j+1}^{n}\left(\begin{array}{c}
k \\
j+1
\end{array}\right) A_{k}(z) \frac{g^{(k-j-1)}(z)}{g(z)}, \quad j=0,1, \ldots, n-2 .
$$

Here $\left(\begin{array}{c}k \\ j+1\end{array}\right)$ denotes the binomial coefficient, and $A_{n}(z) \equiv 1$.

Proof. Set $v=f / g$. By substituting $f=v g$ into equation (6.2), and noting that $u=v^{\prime}$, we deduce the result.

Lemma 6.4. Let $f_{0,1}, f_{0,2}, \ldots, f_{0, m}$ be $m \geq 2$ linearly independent meromorphic solutions of an equation of the form

$$
y^{(n)}+A_{0, n-1}(z) y^{(n-1)}+\cdots+A_{0,0}(z) y=0, \quad n \geq m,
$$

where $A_{0,0}(z), \ldots, A_{0, n-1}(z)$ are meromorphic functions. For $1 \leq q \leq m-1$, set

$$
f_{q, j}=\left(\frac{f_{q-1, j+1}}{f_{q-1,1}}\right)^{\prime}, \quad j=1,2, \ldots, m-q .
$$

Then $f_{q, 1}, f_{q, 2}, \ldots, f_{q, m-q}$ are $m-q$ linearly independent meromorphic solutions of the equation

$$
y^{(n-q)}+A_{q, n-q-1}(z) y^{(n-q-1)}+\cdots+A_{q, 0}(z) y=0,
$$

where

$$
A_{q, j}(z)=\sum_{k=j+1}^{n-q+1}\left(\begin{array}{c}
k \\
j+1
\end{array}\right) A_{q-1, k}(z) \frac{\left(f_{q-1,1}\right)^{(k-j-1)}(z)}{f_{q-1,1}(z)}
$$

for $j=0,1, \ldots, n-q-1$. Here we set $A_{k, n-k}(z) \equiv 1$ for all $k=0,1, \ldots, q$. 
Moreover, suppose that for each $j, j=0,1, \ldots, n-1$, there exists a real number $\tau_{0, j}$ such that

$$
\left|A_{0, j}(z)\right| \leq|z|^{\tau_{0, j}}, \quad|z| \notin E .
$$

Suppose further that each $f_{0, j}$ is of finite order $\rho\left(f_{0, j}\right)$. Set $\beta=\max _{1 \leq j \leq m}\left\{\rho\left(f_{0, j}\right)\right\}$. Then for any given $\varepsilon>0$, we have

$$
\left|A_{q, j}(z)\right| \leq|z|^{\tau_{q, j}}, \quad|z| \notin E,
$$

where

$$
\tau_{q, j}=\max _{q+j \leq k \leq n}\left\{\tau_{0, k}+(k-q-j)(\beta-1)+\varepsilon\right\}
$$

for $j=0,1, \ldots, n-q-1$.

Proof. By applying Lemma 6.2 and Lemma $6.3 q$ times, we obtain (6.4) and (6.5). Therefore, we need only to prove (6.7) and (6.8). For this proof, we use induction on $q$.

First suppose that $q=1$. Then (6.5) is

$$
A_{1, j}(z)=\sum_{k=j+1}^{n}\left(\begin{array}{c}
k \\
j+1
\end{array}\right) A_{0, k}(z) \frac{f_{0,1}^{(k-j-1)}(z)}{f_{0,1}(z)} .
$$

Hence

$$
\left|A_{1, j}(z)\right| \leq \sum_{k=j+1}^{n}\left(\begin{array}{c}
k \\
j+1
\end{array}\right)\left|A_{0, k}(z)\right|\left|\frac{f_{0,1}^{(k-j-1)}(z)}{f_{0,1}(z)}\right| .
$$

Since $\rho\left(f_{0,1}\right) \leq \beta$, it follows from (6.1), (6.6), and (6.10) that (6.7) and (6.8) hold for $q=1$.

For the induction step, we now make the assumption that for any given $\varepsilon>0$, (6.7) and (6.8) hold for $q-1$, i.e.,

$$
\left|A_{q-1, j}(z)\right| \leq|z|^{\tau_{q-1, j}}, \quad|z| \notin E,
$$

where

$$
\tau_{q-1, j}=\max _{q-1+j \leq k \leq n}\left\{\tau_{0, k}+(k-q+1-j)(\beta-1)+\varepsilon\right\}
$$

for $j=0,1, \ldots, n-q$. We now show that (6.7) and (6.8) hold (for $q$ ). From (6.11) and $\rho\left(f_{q-1,1}\right) \leq \beta$, we apply the same argument as above to (6.5), and obtain

$$
\left|A_{q, j}(z)\right| \leq|z|^{\mu_{q, j}}, \quad|z| \notin E,
$$

where

$$
\mu_{q, j}=\max _{j+1 \leq k \leq n-q+1}\left\{\tau_{q-1, k}+(k-j-1)(\beta-1)+\varepsilon\right\} .
$$

From (6.14) and (6.12), we have

$$
\begin{aligned}
\mu_{q, j}= & \max _{j+1 \leq k \leq n-q+1}\left\{\max _{q-1+k \leq l \leq n}\left\{\tau_{0, l}+(l-q+1-k)(\beta-1)+\varepsilon\right\}\right. \\
& +(k-j-1)(\beta-1)+\varepsilon\} \\
\leq & \max _{q+j \leq l \leq n}\left\{\tau_{0, l}+(l-q-j)(\beta-1)+2 \varepsilon\right\} .
\end{aligned}
$$


From (6.15), (6.14), and (6.13), we see that (6.7) and (6.8) hold (for q). This proves the induction step, and therefore completes the proof of Lemma 6.4.

Next we analyze the particular coefficient $A_{q, 0}(z)$ in (6.4), for use in the proof of Lemma 6.5 below. From (6.5), we have

$$
\begin{aligned}
A_{q, 0}= & A_{q-1,1}+\sum_{k=2}^{n-q+1}\left(\begin{array}{l}
k \\
1
\end{array}\right) A_{q-1, k} \frac{f_{q-1,1}^{(k-1)}}{f_{q-1,1}} \\
= & A_{q-2,2}+\sum_{k=3}^{n-q+2}\left(\begin{array}{l}
k \\
2
\end{array}\right) A_{q-2, k} \frac{f_{q-2,1}^{(k-2)}}{f_{q-2,1}}+\sum_{k=2}^{n-q+1}\left(\begin{array}{c}
k \\
1
\end{array}\right) A_{q-1, k} \frac{f_{q-1,1}^{(k-1)}}{f_{q-1,1}} \\
= & \cdots \cdots \\
= & A_{0, q}+\sum_{k=q+1}^{n}\left(\begin{array}{l}
k \\
q
\end{array}\right) A_{0, k} \frac{f_{0,1}^{(k-q)}}{f_{0,1}}+\sum_{k=q}^{n-1}\left(\begin{array}{c}
k \\
q-1
\end{array}\right) A_{1, k} \frac{f_{1,1}^{(k-q+1)}}{f_{1,1}} \\
& \quad+\cdots+\sum_{k=3}^{n-q+2}\left(\begin{array}{l}
k \\
2
\end{array}\right) A_{q-2, k} \frac{f_{q-2,1}^{(k-2)}}{f_{q-2,1}}+\sum_{k=2}^{n-q+1}\left(\begin{array}{c}
k \\
1
\end{array}\right) A_{q-1, k} \frac{f_{q-1,1}^{(k-1)}}{f_{q-1,1}} \\
= & A_{0, q}+\sum_{j=2}^{q+1} H_{j},
\end{aligned}
$$

where

$$
H_{j}(z)=\sum_{k=j}^{n-q+j-1}\left(\begin{array}{c}
k \\
j-1
\end{array}\right) A_{q-j+1, k}(z) \frac{f_{q-j+1,1}^{(k-j+1)}(z)}{f_{q-j+1,1}(z)} .
$$

Then we have the following result.

Lemma 6.5. Under the hypotheses of Lemma 6.4, we have

$$
A_{q, 0}(z)=A_{0, q}(z)+G_{q}(z),
$$

where $G_{q}(z)=\sum_{j=2}^{q+1} H_{j}(z)$ with $H_{j}(z)$ given in (6.17). Moreover, $G_{q}(z)$ satisfies

$$
\left|G_{q}(z)\right| \leq|z|^{\tau_{q}}, \quad|z| \notin E,
$$

where

$$
\tau_{q}=\max _{q+1 \leq k \leq n}\left\{\tau_{0, k}+(k-q)(\beta-1)+\varepsilon\right\} .
$$

Proof. First note that (6.18) is (6.16) with (6.17). Thus we need only to prove (6.19) and (6.20).

Let $j$ be fixed, $2 \leq j \leq q+1$. From (6.17), we have

$$
\left|H_{j}(z)\right| \leq \sum_{k=j}^{n-q+j-1}\left(\begin{array}{c}
k \\
j-1
\end{array}\right)\left|A_{q-j+1, k}(z)\right|\left|\frac{f_{q-j+1,1}^{(k-j+1)}(z)}{f_{q-j+1,1}(z)}\right| .
$$

Since $\rho\left(f_{q-j+1,1}\right) \leq \beta$, we obtain from (6.1), (6.7), and (6.21) that

$$
\left|H_{j}(z)\right| \leq|z|^{\mu_{j}}, \quad|z| \notin E,
$$

where

$$
\mu_{j}=\max _{j \leq k \leq n-q+j-1}\left\{\tau_{q-j+1, k}+(k-j+1)(\beta-1)+\varepsilon\right\} .
$$


However, from (6.23) and (6.8), we have

$$
\begin{aligned}
\mu_{j}= & \max _{j \leq k \leq n-q+j-1}\left\{\max _{q+k-j+1 \leq l \leq n}\left\{\tau_{0, l}+(l-q-k+j-1)(\beta-1)+\varepsilon\right\}\right. \\
& +(k-j+1)(\beta-1)+\varepsilon\} \\
\leq & \max _{q+1 \leq l \leq n}\left\{\tau_{0, l}+(l-q)(\beta-1)+2 \varepsilon\right\} .
\end{aligned}
$$

Since $G_{q}(z)=\sum_{j=2}^{q+1} H_{j}(z),(6.19)$ and (6.20) follow immediately from (6.22), (6.23), and (6.24).

Lemmas 6.6 to 6.9 contain properties of the integers $s_{j}$ and the rational numbers $\alpha_{j}$ in $\S 2$, which we also use in the proof of Theorem 2 .

Lemma 6.6. Suppose that $s_{m-j} \leq k<n$, where $1 \leq j \leq m-1$. Then

$$
d_{s_{m-j-1}}+\left(s_{m-j-1}-k\right)\left(\alpha_{m-j}-1\right) \leq d_{s_{m-j}}+\left(s_{m-j}-k\right)\left(\alpha_{m-j+1}-1\right) .
$$

Proof. If $k=s_{m-j}$, then (6.25) follows directly from the definition of $\alpha_{m-j}$ in (2.4). On the other hand, if $s_{m-j}<k<n$, then using (2.4) we obtain that (6.25) holds

$$
\begin{aligned}
\Longleftrightarrow & \left(s_{m-j-1}-k\right)\left(\alpha_{m-j}-1\right) \leq d_{s_{m-j}}-d_{s_{m-j-1}}+\left(s_{m-j}-k\right)\left(\alpha_{m-j+1}-1\right) \\
\Longleftrightarrow & \left(s_{m-j-1}-k\right)\left(\alpha_{m-j}-1\right) \\
& \leq\left(s_{m-j-1}-s_{m-j}\right)\left(\alpha_{m-j}-1\right)+\left(s_{m-j}-k\right)\left(\alpha_{m-j+1}-1\right) \\
\Longleftrightarrow & \Longleftrightarrow\left(s_{m-j}-k\right)\left(\alpha_{m-j}-1\right) \leq\left(s_{m-j}-k\right)\left(\alpha_{m-j+1}-1\right) \\
\Longleftrightarrow & \alpha_{m-j} \geq \alpha_{m-j+1},
\end{aligned}
$$

which is true from Theorem 1(ii). Lemma 6.6 follows.

Lemma 6.7. Suppose that $s_{m-j} \leq k<n$, where $1 \leq j \leq m-1$. Then

$$
d_{s_{m-j-1}}+\left(s_{m-j-1}-k\right)\left(\alpha_{m-j}-1\right) \leq d_{s_{m-1}}+\left(s_{m-1}-k\right)\left(\alpha_{m}-1\right) \text {. }
$$

Proof. Since $s_{m-j}>s_{m-j+1}>\cdots>s_{m-1}$, applying Lemma 6.6 repeatedly yields

$$
\begin{aligned}
& d_{s_{m-j-1}}+\left(s_{m-j-1}-k\right)\left(\alpha_{m-j}-1\right) \leq d_{s_{m-j}}+\left(s_{m-j}-k\right)\left(\alpha_{m-j+1}-1\right) \\
& \quad \leq d_{s_{m-j+1}}+\left(s_{m-j+1}-k\right)\left(\alpha_{m-j+2}-1\right) \leq \cdots \cdots \\
& \quad \leq d_{s_{m-1}}+\left(s_{m-1}-k\right)\left(\alpha_{m}-1\right) .
\end{aligned}
$$

Lemma 6.8. Suppose that $s_{m}+1 \leq k<n$ for two positive integers $m$ and $k$. Then

$$
d_{k} \leq d_{s_{m-1}}+\left(s_{m-1}-k\right)\left(\alpha_{m}-1\right) .
$$

Proof. If $s_{m}+1 \leq k<s_{m-1}$, then by the definition of $s_{m}$ in (2.2) and (2.3), we have

$$
\frac{d_{k}-d_{s_{m-1}}}{s_{m-1}-k} \leq \frac{d_{s_{m}}-d_{s_{m-1}}}{s_{m-1}-s_{m}}=\alpha_{m}-1 .
$$

Thus (6.26) holds for such $k$.

On the other hand, if $s_{m-1} \leq k<n$, then $m \geq 2$ and $s_{m-j} \leq k<s_{m-j-1}$ for some $j=1,2, \ldots, m-1$. Then by the definition of $s_{m-j}$, we obtain

$$
\frac{d_{k}-d_{s_{m-j-1}}}{s_{m-j-1}-k} \leq \frac{d_{s_{m-j}}-d_{s_{m-j-1}}}{s_{m-j-1}-s_{m-j}}=\alpha_{m-j}-1 \text {. }
$$


Applying Lemma 6.7 to (6.27) gives

$$
d_{k} \leq d_{s_{m-j-1}}+\left(s_{m-j-1}-k\right)\left(\alpha_{m-j}-1\right) \leq d_{s_{m-1}}+\left(s_{m-1}-k\right)\left(\alpha_{m}-1\right),
$$

which is (6.26). Lemma 6.8 is proved.

Lemma 6.9. For all $m=1,2, \ldots, p$, we have

$$
\left(n-s_{m}\right) d_{s_{m-1}} \geq\left(n-s_{m-1}\right) d_{s_{m}} .
$$

Proof. We prove Lemma 6.9 by induction on $m$. Obviously, (6.28) holds for $m=1$, since $s_{0}=n$ and $d_{s_{0}}=d_{n}=0$ from $(2.5)$.

Suppose now that (6.28) holds for $m=j, 1 \leq j \leq p-1$, i.e., suppose that

$$
d_{s_{j-1}} \geq \frac{n-s_{j-1}}{n-s_{j}} d_{s_{j}} .
$$

We will show that (6.28) also holds for $m=j+1$. From the definition of $s_{j}$ in (2.2) and (2.3), we have

$$
\frac{d_{s_{j}}-d_{s_{j-1}}}{s_{j-1}-s_{j}}>\frac{d_{s_{j+1}}-d_{s_{j-1}}}{s_{j-1}-s_{j+1}}
$$

Hence

$$
\left(s_{j-1}-s_{j+1}\right) d_{s_{j}}>\left(s_{j-1}-s_{j}\right) d_{s_{j+1}}+\left(s_{j}-s_{j+1}\right) d_{s_{j-1}} .
$$

Substituting (6.29) into (6.30) and simplifying gives

$$
\left(s_{j-1}-s_{j}\right)\left(n-s_{j+1}\right) d_{s_{j}}>\left(s_{j-1}-s_{j}\right)\left(n-s_{j}\right) d_{s_{j+1}},
$$

which means (6.28) holds for $m=j+1$, since the common factor $s_{j-1}-s_{j}(>0)$ on both sides can be deleted. This proves the induction step, and completes the proof of Lemma 6.9 .

Remark. As mentioned in $\S 2$, we now give a new proof of Theorem 1(iii) by using Lemma 6.1. We show that if $s_{1}=0$, then every nontrivial solution of (1.1) has order $1+d_{0} / n$.

Suppose to the contrary that there exists a nontrivial solution $f$ of (1.1) which satisfies $\rho(f)<1+d_{0} / n$. Set $\beta=\rho(f)$. Then

$$
\beta=1+\frac{d_{0}}{n}-\tau,
$$

where $\tau$ is a positive constant. We will show that this results in a contradiction.

Since $s_{1}=0$, from $(2.2)$ we have

$$
d_{k} \leq \frac{n-k}{n} d_{0}, \quad k=1,2, \ldots, n-1 .
$$

Since $f$ is a solution of $(1.1)$, we obtain

$$
-p_{0}(z)=\frac{f^{(n)}}{f}+p_{n-1}(z) \frac{f^{(n-1)}}{f}+\cdots+p_{1}(z) \frac{f^{\prime}}{f},
$$

from which it follows that

$$
\left|p_{0}(z)\right| \leq \sum_{k=1}^{n}\left|p_{k}(z) \frac{f^{(k)}(z)}{f(z)}\right|
$$

where we set $p_{n}(z) \equiv 1$. 
Since $p_{k}(z)$ is a polynomial of degree $d_{k}$ and $f$ is of order $\beta$, from (6.1) and (6.33) we obtain that for any given $\varepsilon>0$,

$$
\left|p_{0}(z)\right| \leq \sum_{k=1}^{n}|z|^{d_{k}+k(\beta-1)+2 \varepsilon}, \quad|z| \notin E .
$$

From (6.34), (6.32), and (6.31), we have

$$
\left|p_{0}(z)\right| \leq \sum_{k=1}^{n}|z|^{d_{0}-k \tau+2 \varepsilon} \leq n|z|^{d_{0}-\tau+2 \varepsilon}, \quad|z| \notin E,
$$

which is impossible if we choose $2 \varepsilon<\tau$, since $d_{0}=\operatorname{deg} p_{0}(z)$. This proves Theorem 1(iii).

\section{Proof of Theorem 2}

Assume the contrary, i.e., suppose that for some integer $m$ satisfying $1 \leq m \leq p$, an equation of the form (1.1) admits $s_{m}+1$ linearly independent solutions with order less than $\alpha_{m}$. We show that this assumption results in a contradiction.

We consider two separate cases.

Case (i). Suppose that $s_{m} \geq 1$.

We denote these $s_{m}+1$ linearly independent solutions of (1.1) by $f_{0,1}, f_{0,2}, \ldots$, $f_{0, s_{m}+1}$, and we define $\beta$ to be the maximum order of these $s_{m}+1$ solutions. Then our assumption is that

$$
\beta=\max _{1 \leq k \leq s_{m}+1}\left\{\rho\left(f_{0, k}\right)\right\}<\alpha_{m} .
$$

Denote $A_{0, k}(z)=p_{k}(z)$ for $k=0,1, \ldots, n-1$. We now perform the method of reduction of order on equation (1.1), by using these solutions $\left\{f_{0, j}\right\}$ of (1.1). As in Lemma 6.4 , for $1 \leq q \leq s_{m}$, set

$$
f_{q, j}=\left(\frac{f_{q-1, j+1}}{f_{q-1,1}}\right)^{\prime}, \quad j=1,2, \ldots, s_{m}+1-q .
$$

From Lemma $6.4, f_{q, 1}, f_{q, 2}, \ldots, f_{q, s_{m}+1-q}$ are linearly independent meromorphic solutions of equation (6.4). Taking $q=s_{m}$ and using (7.2) and Lemma 6.4, we obtain that the function $f=f_{s_{m}, 1}(z)$ is a nontrivial solution of an equation of the form

$$
f^{\left(n-s_{m}\right)}+A_{s_{m}, n-s_{m}-1}(z) f^{\left(n-s_{m}-1\right)}+\cdots+A_{s_{m}, 0}(z) f=0,
$$

where the coefficients $A_{s_{m}, n-s_{m}-1}(z), \ldots, A_{s_{m}, 0}(z)$ are meromorphic functions which for any given $\varepsilon>0$ satisfy

$$
\left|A_{s_{m}, j}(z)\right| \leq|z|^{\tau_{s_{m}, j}}, \quad|z| \notin E,
$$

where

$$
\tau_{s_{m}, j}=\max _{s_{m}+j \leq k \leq n}\left\{d_{k}+\left(k-s_{m}-j\right)(\beta-1)+\varepsilon\right\}
$$

for $j=0,1, \ldots, n-s_{m}-1$, and $d_{k}=\operatorname{deg} p_{k}(z)=\operatorname{deg} A_{0, k}(z)$.

Note that $\rho\left(f_{s_{m}, 1}\right) \leq \beta$. Hence it follows from (7.4), (7.5), and (6.1) that for $j=0,1, \ldots, n-s_{m}-1$,

$$
\left|A_{s_{m}, j}(z) \frac{f_{s_{m}, 1}^{(j)}(z)}{f_{s_{m}, 1}(z)}\right| \leq|z|^{\sigma_{j}}, \quad|z| \notin E,
$$


where

$$
\sigma_{j}=\max _{s_{m}+j \leq k \leq n}\left\{d_{k}+\left(k-s_{m}\right)(\beta-1)+2 \varepsilon\right\} .
$$

However, from (7.3) we have

$$
-A_{s_{m}, 0}(z)=\frac{f_{s_{m}, 1}^{\left(n-s_{m}\right)}}{f_{s_{m}, 1}}+\sum_{j=1}^{n-s_{m}-1} A_{s_{m}, j}(z) \frac{f_{s_{m}, 1}^{(j)}}{f_{s_{m}, 1}}
$$

Therefore, from (7.6), (7.7), and (7.8), we obtain

$$
\left|A_{s_{m}, 0}(z)\right| \leq|z|^{\eta}, \quad|z| \notin E
$$

where

$$
\eta=\max _{s_{m}+1 \leq k \leq n}\left\{d_{k}+\left(k-s_{m}\right)(\beta-1)+2 \varepsilon\right\} .
$$

However, from Lemma 6.5, we have

$$
A_{0, s_{m}}(z)=A_{s_{m}, 0}(z)-G_{s_{m}}(z),
$$

where $G_{s_{m}}(z)$ satisfies (6.19) and (6.20) with $q$ replaced by $s_{m}$. Hence from (7.9), (7.11), (6.19), and (6.20) (with $q=s_{m}$ ), we obtain

$$
\left|A_{0, s_{m}}(z)\right| \leq|z|^{\eta}, \quad|z| \notin E
$$

where $\eta$ is the number in (7.10). Here, note that $\tau_{0, k}=d_{k}+\varepsilon$ in (6.20).

Finally, we will show that (7.12) results in a contradiction. To this end, we will prove that, for any $k$ satisfying $s_{m}+1 \leq k \leq n$,

$$
d_{k}+\left(k-s_{m}\right)(\beta-1) \leq d_{s_{m}}-\alpha,
$$

where $\alpha=\alpha_{m}-\beta>0$ from (7.1). Once (7.13) has been established, then a contradiction will follow immediately, since from (7.13), (7.12), and (7.10) we obtain

$$
\left|A_{0, s_{m}}(z)\right| \leq|z|^{d_{s_{m}}-\alpha+2 \varepsilon}
$$

which is impossible when $2 \varepsilon<\alpha$, because $A_{0, s_{m}}(z)=p_{s_{m}}(z)$ is a polynomial of degree $d_{s_{m}}$.

To prove (7.13), we will use Lemmas 6.8 and 6.9. We consider the cases $s_{m}+1 \leq$ $k<n$ and $k=n$ separately.

If $s_{m}+1 \leq k<n$, then from Lemma 6.8 and the definition of $\alpha_{m}$ in (2.4), we obtain

$$
\begin{aligned}
d_{k}+\left(k-s_{m}\right)(\beta-1) & \leq d_{s_{m-1}}+\left(s_{m-1}-k\right)\left(\alpha_{m}-1\right)+\left(k-s_{m}\right)(\beta-1) \\
& =d_{s_{m}}-\alpha\left(k-s_{m}\right) \\
& \leq d_{s_{m}}-\alpha .
\end{aligned}
$$

On the other hand, from Lemma 6.9 , we obtain

$$
\left(n-s_{m}\right)\left(d_{s_{m}}-d_{s_{m-1}}\right) \leq\left(s_{m-1}-s_{m}\right) d_{s_{m}} .
$$

Noting that $d_{n}=0$, this gives

$$
\begin{aligned}
d_{n}+\left(n-s_{m}\right)(\beta-1) & =\left(n-s_{m}\right)\left(\alpha_{m}-1\right)-\alpha\left(n-s_{m}\right) \\
& \leq\left(n-s_{m}\right) \frac{d_{s_{m}}-d_{s_{m-1}}}{s_{m-1}-s_{m}}-\alpha \\
& \leq d_{s_{m}}-\alpha .
\end{aligned}
$$


Therefore, from (7.15) and (7.14), we see that (7.13) holds for all $s_{m}+1 \leq k \leq n$. This proves (7.13), which completes the proof of Case (i).

Case (ii). Suppose that $s_{m}=0$.

In this case, $m=p$ and $s_{p}=0$. We thus need to show that equation (1.1) does not admit a nontrivial solution with order less than $\alpha_{p}$.

To this end, we assume that there exists a solution $f_{0} \not \equiv 0$ of $(1.1)$ with $\rho\left(f_{0}\right)<$ $\alpha_{p}$. Then, by Theorem 1(i), $f_{0}$ must be a polynomial.

From (1.1), we obtain

$$
-p_{0}(z)=p_{1}(z) \frac{f_{0}^{\prime}}{f_{0}}+\cdots+p_{n-1}(z) \frac{f_{0}^{(n-1)}}{f_{0}}+\frac{f_{0}^{(n)}}{f_{0}} .
$$

Since $f_{0}$ is a polynomial, it follows from (7.16) that

$$
d_{0} \leq \max _{1 \leq k \leq n-1}\left\{d_{k}-k\right\}
$$

Applying Lemma 6.8 with $m=p$ gives

$$
d_{k} \leq d_{s_{p-1}}+\left(s_{p-1}-k\right)\left(\alpha_{p}-1\right)
$$

for all $1 \leq k \leq n-1$, since $s_{p}=0$. Therefore, from (7.18), the definition of $\alpha_{p}$ in (2.4), and the fact that $s_{p}=0$, we obtain for any $1 \leq k \leq n-1$,

$$
\begin{aligned}
d_{k}-k & \leq d_{s_{p-1}}-k+\left(s_{p-1}-k\right)\left(\alpha_{p}-1\right) \\
& =d_{0}+\frac{k}{s_{p-1}}\left(d_{s_{p-1}}-d_{0}-s_{p-1}\right) .
\end{aligned}
$$

Since $\alpha_{p}>0$ and $s_{p}=0$, it follows from the definition of $\alpha_{p}$ in (2.4) that $d_{s_{p-1}}<$ $s_{p-1}+d_{0}$. Hence from $(7.19)$, we obtain $d_{k}-k<d_{0}$ for all $k=1,2, \ldots, n-1$. But this contradicts (7.17).

This proves Case (ii), and thus completes the proof of Theorem 2.

\section{EXAMPLES}

In this section we give several examples which illustrate the sharpness of our results and which also exhibit some possibilities that can occur.

Example 1. We now exhibit a special contour integral solution $f=G(z)$ of the third order differential equation

$$
f^{\prime \prime \prime}-z f^{\prime \prime}-f=0
$$

which satisfies $\rho(G)=1 / 2$. This gives equality in (2.7) when $n=3$.

We define the function $G(z)$ as follows:

$$
G(z)=\int_{K_{R}} \exp \left\{\frac{z}{w}-\frac{1}{2 w^{2}}-w\right\} d w, \quad \text { where } \quad K_{R}:|w|=R \quad(R>0)
$$

For any fixed $z$, the integrand in (8.2) is analytic for all $w \neq 0$. From Cauchy's theorem it follows that for any fixed $z$, the value of $G(z)$ is independent of $R>0$. 
From (8.2) we obtain

$$
\begin{aligned}
G^{\prime \prime \prime}(z)-z G^{\prime \prime}(z)-G(z) & =\int_{K_{R}}\left(\frac{1}{w^{3}}-\frac{z}{w^{2}}-1\right) \exp \left\{\frac{z}{w}-\frac{1}{2 w^{2}}-w\right\} d w \\
& =\int_{K_{R}} \frac{\partial}{\partial w} \exp \left\{\frac{z}{w}-\frac{1}{2 w^{2}}-w\right\} d w \\
& =\left[\exp \left\{\frac{z}{w}-\frac{1}{2 w^{2}}-w\right\}\right]_{K_{R}}=0,
\end{aligned}
$$

since $K_{R}$ is a closed curve. Thus $f=G(z)$ is a solution of equation (8.1).

Now note that equation (8.1) cannot possess a nontrivial polynomial solution. Hence it follows from Theorem 1(i) that we must have exactly one of the following:

$$
\rho(G)=2, \quad \rho(G)=1 / 2, \quad \text { or } \quad G \equiv 0 .
$$

We will show that $\rho(G)=1 / 2$.

We first prove that $G \not \equiv 0$. To prove this, we show that $G^{\prime}(0) \neq 0$. Since the value of $G(z)$ is independent of $R>0$ in (8.2), we use $R=1$ and obtain

$$
G^{\prime}(0)=\int_{K_{1}} \frac{1}{w} \exp \left\{-\frac{1}{2 w^{2}}-w\right\} d w=\int_{-\pi}^{\pi} i \exp \left\{-\frac{1}{2 e^{i 2 \theta}}-e^{i \theta}\right\} d \theta .
$$

We break this expression into real and imaginary parts. The real part of the integrand is an odd function, while the imaginary part of the integrand is an even function. This gives

$$
G^{\prime}(0)=2 i \int_{0}^{\pi} \exp \left\{-\frac{\cos 2 \theta}{2}-\cos \theta\right\} \cos L(\theta) d \theta,
$$

where

$$
L(\theta)=\frac{\sin 2 \theta}{2}-\sin \theta
$$

Consider now the function $L(\theta)$ in (8.6). Since

$$
L^{\prime}(\theta)=\cos 2 \theta-\cos \theta=2 \cos ^{2} \theta-1-\cos \theta=(2 \cos \theta+1)(\cos \theta-1),
$$

we obtain $L^{\prime}(\theta)<0$ for $0<\theta<\frac{2 \pi}{3}$ and $L^{\prime}(\theta)>0$ for $\frac{2 \pi}{3}<\theta<\pi$. Also, $L(0)=L(\pi)=0$ and $L\left(\frac{2 \pi}{3}\right)<0$. Hence, $L(\theta)<0$ for $0<\theta<\pi$. More specifically, we have

$$
-\frac{\pi}{2}<-\frac{3 \sqrt{3}}{4}=L\left(\frac{2 \pi}{3}\right) \leq L(\theta)<0 \quad \text { for } \quad 0<\theta<\pi,
$$

which implies that $\cos L(\theta)>0$ for $0<\theta<\pi$. Then from (8.5) we obtain that $G^{\prime}(0) \neq 0$. Hence

$$
G \not \equiv 0 .
$$

We next show that $\rho(G) \leq 1 / 2$. To see this, let $z \neq 0$ be fixed, and choose $R=\sqrt{|z|}>0$ in (8.2), i.e., $K_{R}:|w|=\sqrt{|z|}$. Then

$$
|G(z)| \leq \int_{K_{R}} \exp \left\{\frac{|z|}{|w|}+\frac{1}{2|w|^{2}}+|w|\right\}|d w|=2 \pi \sqrt{|z|} \exp \left\{2 \sqrt{|z|}+\frac{1}{2|z|}\right\} .
$$

By letting $z \rightarrow \infty$, we see that $\rho(G) \leq 1 / 2$. By combining this fact with (8.7) and (8.3), we obtain that $\rho(G)=1 / 2$. Since $f=G(z)$ satisfies equation (8.1), this is an example where the inequality (2.7) becomes an equality in the case when $n=3$. 
We also mention the following observation. By differentiating equation (8.1) and then adding the resulting equation to (8.1), we obtain that $f=G(z)$ is a solution of the fourth order equation

$$
f^{(4)}+(1-z) f^{\prime \prime \prime}-(1+z) f^{\prime \prime}-f^{\prime}-f=0 .
$$

Continuing in this manner, we see that for any $n \geq 3$ we can obtain a particular equation of the form (1.1) that possesses a solution of order $1 / 2$, namely, $f=G(z)$.

Remark. For $n \geq 2$, consider an equation of the form

$$
f^{(n)}+p(z) f^{(n-1)}+c_{n-2} f^{(n-2)}+\cdots+c_{1} f^{\prime}+c_{0} f=0,
$$

where $c_{0}, c_{1}, \ldots, c_{n-2}$ are all constants $\left(c_{0} \neq 0\right)$ and $p(z)$ is a polynomial of degree $n-2$. From Theorem 1(i), there are two possible orders $\alpha_{1}=n-1$ and $\alpha_{2}=$ $1 /(n-1)$ of transcendental solutions of equation (8.8). Wittich [8], [9, pp. 65-68] indicates that there always exists a solution $f=\psi(z)$ of equation (8.8) that satisfies $\rho(\psi)=1 /(n-1)$, which gives an equality in (2.7) for all $n \geq 2$.

Examples 2 through 6 below illustrate the sharpness of Corollary 1, Theorem 2, and Corollary 5, and are also examples of Theorem 1.

Example 2. Let $q_{1}, q_{2}, \ldots, q_{n}$ be any $n$ distinct integers that satisfy

$$
0<q_{1}<q_{2}<\ldots<q_{n} .
$$

We construct an equation of the form (1.1) which possesses $n$ distinct solutions $f_{1}, f_{2}, \ldots, f_{n}$ satisfying $\rho\left(f_{k}\right)=q_{k}$ for $1 \leq k \leq n$. This illustrates the sharpness of Corollary 1 and Theorem 2. Furthermore, the equation of the form (1.1) that we construct satisfies $d_{0}=\operatorname{deg} p_{0}=q_{1}+q_{2}+\cdots+q_{n}-n$, which means that this is a sharp example for Corollary 5 , because $(2.11)$ becomes an equality $\left(=q_{1}+q_{2}+\cdots+q_{n}\right)$.

We construct the equation as follows. Set

$$
A_{1,0}(z)=-q_{1} z^{q_{1}-1} .
$$

Then $f_{1}(z)=\exp \left(z^{q_{1}}\right)$ is a solution of the equation

$$
L_{1}(f)=f^{\prime}+A_{1,0}(z) f=0,
$$

and $\rho\left(f_{1}\right)=q_{1}$.

Since $f_{1}$ is a solution of $(8.9), f_{1}$ is also a solution of the two equations

$$
f^{\prime \prime}+A_{1,0}(z) f^{\prime}+A_{1,0}^{\prime}(z) f=0
$$

and

$$
z^{q_{2}-1} f^{\prime}+z^{q_{2}-1} A_{1,0}(z) f=0 .
$$

By adding (8.10) and (8.11), we obtain that $f_{1}$ is a solution of the equation

$$
L_{2}(f)=f^{\prime \prime}+A_{2,1}(z) f^{\prime}+A_{2,0}(z) f=0,
$$

where $A_{2,1}(z)=z^{q_{2}-1}+A_{1,0}(z)$ and $A_{2,0}(z)=z^{q_{2}-1} A_{1,0}(z)+A_{1,0}^{\prime}(z)$. Note that

$$
L_{2}(f)=z^{q_{2}-1} L_{1}(f)+\frac{d}{d z}\left(L_{1}(f)\right) .
$$

Since $0<q_{1}<q_{2}, A_{2,1}(z)$ is a polynomial of degree $q_{2}-1$. We also note that $\operatorname{deg} A_{2,0}=q_{1}+q_{2}-2$. Now let $f_{2}$ be any solution of (8.12) such that $f_{1}$ and $f_{2}$ 
are linearly independent. From (8.12) and Abel's identity [4, p.16], the Wronskian $W\left(f_{1}, f_{2}\right)$ satisfies

$$
W\left(f_{1}, f_{2}\right)=C \exp \left(\int-A_{2,1}(z) d z\right)
$$

for some constant $C \neq 0$. Since $\rho\left(f_{1}\right)=q_{1}$ and $\rho\left(W\left(f_{1}, f_{2}\right)\right)=1+\operatorname{deg} A_{2,1}=q_{2}>$ $q_{1}$, we conclude that $\rho\left(f_{2}\right) \geq q_{2}$. However, from (1.2) and (1.3), we have

$$
\rho\left(f_{2}\right) \leq 1+\max \left\{\operatorname{deg} A_{2,1}, \frac{\operatorname{deg} A_{2,0}}{2}\right\}=1+\max \left\{q_{2}-1, \frac{q_{1}+q_{2}-2}{2}\right\}=q_{2} .
$$

Hence $\rho\left(f_{2}\right)=q_{2}$. (Note: Alternatively, it also follows from Corollary 3 that we must have $\rho\left(f_{2}\right)=q_{2}$.) Therefore, $f_{1}$ and $f_{2}$ are solutions of equation (8.12) such that $\rho\left(f_{k}\right)=q_{k}$ for $k=1,2$.

Continuing in this manner, we can contruct the desired general example. Namely, we define

$$
L_{j}(f)=z^{q_{j}-1} L_{j-1}(f)+\frac{d}{d z}\left(L_{j-1}(f)\right)
$$

for $3 \leq j \leq n$. Then by the same argument as above, it can be shown that the $j$-th order linear differential equation $L_{j}(f)=0$ admits $j$ solutions $f_{1}, f_{2}, \ldots, f_{j}$ such that $\rho\left(f_{k}\right)=q_{k}$ for $k=1,2, \ldots, j$. Furthermore, the coefficient of $f$ in the differential equation $L_{j}(f)=0$ is a polynomial of degree $q_{1}+q_{2}+\cdots+q_{j}-j$.

Example 3. The equation

$$
f^{\prime \prime \prime}-f^{\prime \prime}+z f^{\prime}-z f=0
$$

possesses the solution $f_{1}=e^{z}$, and, by Corollary 3 , a solution $f_{2}$ satisfying $\rho\left(f_{2}\right)=$ $3 / 2$. For this equation we have $p=2, s_{1}=1, s_{2}=0, \alpha_{1}=3 / 2$, and $\alpha_{2}=1$. This gives a sharp example for Corollary 1 and Theorem 2 .

It can also be deduced that this gives a sharp example for Corollary 5 . Specifically, since $\alpha_{1}=3 / 2$ and $\alpha_{2}=1$, it follows from Theorem 2 or Corollary 5 that there exists a third solution $f_{3}$ of $(8.13)$ that satisfies $\rho\left(f_{3}\right)=3 / 2$, such that $\left\{f_{1}, f_{2}, f_{3}\right\}$ is a fundamental set of solutions of (8.13). Hence for the fundamental solution set $\left\{f_{1}, f_{2}, f_{3}\right\}$, we obtain the equality $4=4$ in (2.11).

Example 4. The equation

$$
f^{(4)}-f^{\prime \prime \prime}+z f^{\prime}-z f=0
$$

possesses the solution $f_{1}=e^{z}$, and, by Corollary 3 , a solution $f_{2}$ satisfying $\rho\left(f_{2}\right)=$ $4 / 3$. For this equation we have $p=2, s_{1}=1, s_{2}=0, \alpha_{1}=4 / 3$, and $\alpha_{2}=1$. This gives a sharp example for Corollary 1 and Theorem 2 . By reasoning similar to that in Example 3, we can deduce that this also gives a sharp example for Corollary 5, because we can obtain the equality $5=5$ in (2.11).

Example 5. The equation

$$
\begin{aligned}
f^{(4)} & +\left(8 z^{3}-13\right) f^{\prime \prime}-\left(16 z^{4}+16 z^{3}+12 z^{2}+4 z+2\right) f^{\prime} \\
& +\left(16 z^{4}+8 z^{3}+12 z^{2}+4 z+14\right) f=0
\end{aligned}
$$

possesses the solutions $f_{1}=e^{z}$ and $f_{2}=e^{z^{2}}$, and, by Corollary 3 , a solution $f_{3}$ satisfying $\rho\left(f_{3}\right)=5 / 2$. For this equation we have $p=3, s_{1}=2, s_{2}=1, s_{3}=$ $0, \alpha_{1}=5 / 2, \alpha_{2}=2$, and $\alpha_{3}=1$. This gives a sharp example for Corollary 1 , Theorem 2, and (by reasoning similar to that in Example 3) Corollary 5. 
Example 6. Let $q$ and $n$ be integers satisfying $2 \leq q \leq n$, and consider the equation

$$
\begin{aligned}
f^{(n)}-\frac{1}{(q-1) !} z^{q-1} f^{(q-1)}+\frac{1}{(q-2) !} z^{q-2} f^{(q-2)}-\cdots & \\
& +(-1)^{q-1} z f^{\prime}+(-1)^{q} f=0 .
\end{aligned}
$$

Observe that equation (8.14) has the $q-1$ linearly independent polynomial solutions $f_{k}(z)=z^{k}, k=1,2, \ldots, q-1$. For equation (8.14), we have $p=1, s_{1}=q-1$, and $\alpha_{1}=n /(n-q+1)$. Therefore, we see that equation (8.14) gives a sharp example for Theorem 2 and Corollary 4.

Moreover, from Theorem 2 or Corollary 5 , we deduce that there exist $n-q+1$ linearly independent solutions $g_{1}, g_{2}, \ldots, g_{n-q+1}$ of (8.14) that satisfy $\rho\left(g_{j}\right)=\alpha_{1}=$ $n /(n-q+1)$ for each $1 \leq j \leq n-q+1$, and that $\left\{g_{1}, g_{2}, \ldots, g_{n-q+1}, z, z^{2}, \ldots, z^{q-1}\right\}$ forms a fundamental set of solutions of (8.14). Hence equation (8.14) also gives a sharp example for Corollary 5.

In the special case when $q=n$, equation (8.14) is an equation of order $n$ that possesses $n-1$ linearly independent polynomial solutions and a transcendental solution of order $n$.

Example 1 is an example of an equation of the form (1.1) that possesses a special contour integral solution of order $1 / 2$. The next example is another equation of the form (1.1) that possesses a special transcendental contour integral solution with order less than one.

Example 7. We now show that there exists a special contour integral solution $f=H(z)$ of the fourth order equation

$$
f^{(4)}-z f^{\prime \prime \prime}-f=0
$$

which satisfies $\rho(H)=2 / 3$. Then by the technique given at the end of Example 1 , we see that for any $n \geq 4$, there will exist an equation of the form (1.1) that possesses a solution of order $2 / 3$.

We define the function $H(z)$ as follows:

$$
H(z)=\int_{C} \exp \left\{\frac{z}{\sqrt{2 w}}-\frac{1}{4 w}-w\right\} d w
$$

where $C$ is the contour defined by $C=C_{1}+C_{2}+C_{3}$ with

$$
\begin{array}{ll}
C_{1}: & w=r e^{i \pi / 4}, r \text { goes from }+\infty \text { to } 1, \\
C_{2}: & w=e^{i \theta}, \theta \text { goes from } \pi / 4 \text { to } 7 \pi / 4, \\
C_{3}: & w=r e^{i 7 \pi / 4}, r \text { goes from } 1 \text { to }+\infty,
\end{array}
$$

and where $\sqrt{2 w}$ is defined by the branch $\sqrt{\zeta}=\exp \left\{\frac{1}{2} \log |\zeta|+i \frac{1}{2} \arg \zeta\right\}, 0<\arg \zeta<$ $2 \pi$.

From (8.16),

$$
\begin{aligned}
H^{(4)}(z)-z H^{\prime \prime \prime}(z)-H(z) & =\int_{C}\left(\frac{1}{4 w^{2}}-\frac{z}{(2 w)^{3 / 2}}-1\right) \exp \left\{\frac{z}{\sqrt{2 w}}-\frac{1}{4 w}-w\right\} d w \\
& =\left[\exp \left\{\frac{z}{\sqrt{2 w}}-\frac{1}{4 w}-w\right\}\right]_{C}=0 .
\end{aligned}
$$

Thus $f=H(z)$ is a solution of equation (8.15). 
Since equation (8.15) cannot possess a nontrivial polynomial solution, it follows from Theorem 1(i) that we must have exactly one of the following:

$$
\rho(H)=2, \quad \rho(H)=2 / 3, \quad \text { or } \quad H \equiv 0 .
$$

We will show that $\rho(H)=2 / 3$.

We will first show that $H \not \equiv 0$ by calculating that $H(0) \neq 0$. From (8.16),

$$
H(0)=\int_{C} \exp \left\{-\frac{1}{4 w}-w\right\} d w
$$

By appealing to Cauchy's theorem, it can be deduced that the contour $C$ in (8.18) can be replaced by the unit circle $|w|=1$. Then, from the residue theorem, we obtain

$$
H(0)=\int_{|w|=1} \exp \left\{-\frac{1}{4 w}-w\right\} d w=2 \pi i \operatorname{Res}(g(w), 0),
$$

where

$$
g(w)=\exp \left\{-\frac{1}{4 w}-w\right\}=\sum_{k=0}^{\infty} \frac{\left(-\frac{1}{4 w}-w\right)^{k}}{k !} .
$$

From inspection of (8.20), we see that the even terms in the series contribute only even powers of $w$ and thus contribute 0 to $\operatorname{Res}(g(w), 0)$, while the odd terms in the series contribute only odd powers of $w$ with negative constant factors. Therefore, $\operatorname{Res}(g(w), 0)<0$. Hence from (8.19), $H(0) \neq 0$. Thus

$$
H \not \equiv 0 .
$$

We next show that $\rho(H) \leq 2 / 3$. By appealing to Cauchy's theorem, it can be seen that we can replace the contour $C$ in (8.16) with the following curve $\gamma_{R}, R>1$ : $\gamma_{R}=K_{1}+K_{2}+K_{3}$, where

$$
\begin{array}{ll}
K_{1}: & w=x+i, x \text { goes from }+\infty \text { to } \sqrt{R^{2}-1}, \\
K_{2}: & w=R e^{i \theta}, \theta \text { goes from } \arctan \left(\frac{1}{\sqrt{R^{2}-1}}\right) \text { to } 2 \pi-\arctan \left(\frac{1}{\sqrt{R^{2}-1}}\right), \\
K_{3}: & w=x-i, x \text { goes from } \sqrt{R^{2}-1} \text { to }+\infty .
\end{array}
$$

Then

$$
H(z)=\int_{\gamma_{R}} \exp \left\{\frac{z}{\sqrt{2 w}}-\frac{1}{4 w}-w\right\} d w
$$

where for a fixed value of $z$, the value of $H(z)$ is independent of $R>1$.

Now for any fixed $z$ satisfying $|z|>1$, we choose $R=|z|^{2 / 3}$. Then

$$
|H(z)| \leq \int_{\gamma_{R}} \exp \left\{\frac{|z|}{\sqrt{2|w|}}+\frac{1}{4|w|}\right\}\left|e^{-w}\right||d w| \leq \exp \left\{\frac{|z|^{2 / 3}}{\sqrt{2}}+\frac{1}{4}\right\} \int_{\gamma_{R}}\left|e^{-w}\right||d w|
$$

and

$$
\int_{\gamma_{R}}\left|e^{-w}\right||d w|<e^{|z|^{2 / 3}} \cdot 2 \pi|z|^{2 / 3}+2 \int_{1}^{\infty} e^{-x} d x
$$

It follows that $\rho(H) \leq 2 / 3$. Therefore, from (8.21) and (8.17), we obtain that $\rho(H)=2 / 3$. Since $f=H(z)$ satisfies (8.15) and $\rho(H)=2 / 3$, this proves the assertion. 
Example 8. Theorem 1 gives all the possible orders $\alpha_{1}, \alpha_{2}, \ldots, \alpha_{p}$ of transcendental solutions of equation (1.1). This example shows that not every value $\alpha_{j}, j=$ $1,2, \ldots, p$, is necessarily attained as the order of a solution of (1.1). Consider the equation

$$
f^{\prime \prime}+z^{3} f^{\prime}-\left(z^{4}+z^{2}\right) f=0 .
$$

Here $p=2, \alpha_{1}=4$, and $\alpha_{2}=2$. So 4 and 2 are the only possible orders for transcendental solutions of (8.22). Clearly, (8.22) does not possess a nontrivial polynomial solution. Moreover, if $f$ is any transcendental solution of (8.22), and if we set

$$
v=f e^{-\frac{1}{2} z^{2}}
$$

then $v$ is a solution of the equation

$$
v^{\prime \prime}+\left(z^{3}+2 z\right) v^{\prime}+v=0
$$

Obviously, (8.24) does not admit a nontrivial polynomial solution. From Theorem 1(i), all transcendental solutions of (8.24) have order 4. Hence from (8.24) and (8.23), we conclude that $\rho(f)=4$. Therefore, all solutions $f \not \equiv 0$ of (8.22) satisfy $\rho(f)=\alpha_{1}=4$, and (8.22) does not admit any solution with order $\alpha_{2}=2$.

Example 9. From Theorem 1(iii), if

$$
\frac{\operatorname{deg} p_{k}}{n-k} \leq \frac{\operatorname{deg} p_{0}}{n}
$$

holds for all $k=1,2, \ldots, n-1$, then all solutions $f \not \equiv 0$ of $(1.1)$ satisfy $\rho(f)=\lambda=$ $1+\operatorname{deg} p_{0} / n$. Example 8 shows that (8.25) is not a necessary condition to ensure that all solutions of (1.1) have the maximum possible order $\lambda$; see (1.2) and (1.3). Here is another example.

Consider the equation

$$
f^{\prime \prime \prime}+z^{5} f^{\prime \prime}+z^{2} f^{\prime}+z^{2} f=0
$$

Here we have $p=1$ and $\alpha_{1}=\lambda=6$. Hence, by Theorem 1(i), all transcendental solutions of (8.26) have order $\lambda$. Clearly, (8.26) does not have a nontrivial polynomial solution. Therefore, all nontrivial solutions of (8.26) have order $\lambda=6$, and equation (8.26) does not satisfy the condition (8.25).

\section{REFERENCES}

1. G. Gundersen, Estimates for the logarithmic derivative of a meromorphic function, plus similar estimates, J. London Math. Soc. 37 (1988), 88-104. MR 88m:30076

2. W. Helmrath and J. Nikolaus, Ein elementarer Beweis bei der Anwendung der Zentralindexmethode auf Differentialgleichungen, Complex Variables 3 (1984), 387-396. MR 86a:30045

3. G. Jank and L. Volkmann, Einführung in die Theorie der ganzen und meromorphen Funktionen mit Anwendungen auf Differentialgleichungen, Birkhäuser, Basel-Boston-Stuttgart, 1985. MR 87h:30066

4. I. Laine, Nevanlinna Theory and Complex Differential Equations, Walter de Gruyter, BerlinNew York, 1993. MR 94d:34008

5. K. Pöschl, Über Anwachsen und Nullstellenverteilung der ganzen transzendenten Lösungen linearer Differentialgleichungen, I, J. Reine Angew. Math. 199 (1958), 121-138. MR 20:6561

6. G. Valiron, Lectures on the general theory of integral functions, translated by E. F. Collingwood, Chelsea, New York, 1949.

7. A. Wiman, Über den Zusammenhang zwischen dem Maximalbetrage einer analytischen Funktion und dem größten Betrage bei gegebenem Argumente der Funktion, Acta Math. 41 (1916), $1-28$. 
8. H. Wittich, Über das Anwachsen der Lösungen linearer Differentialgleichungen, Math. Ann. 124 (1952), 277-288. MR 14:171d

9. H. Wittich, Neuere Untersuchungen über eindeutige analytische Funktionen, 2nd Edition, Springer-Verlag, Berlin-Heidelberg-New York, 1968. MR 39:5804

Department of Mathematics, University of New Orleans, New Orleans, Louisiana 70148

E-mail address: ggunders@math.uno.edu

E-mail address: esteinba@math.uno.edu

E-mail address: swang@math.uno.edu 